\title{
Evidence for nodal superconductivity in a layered compound $\operatorname{Ta}_{4} \operatorname{Pd}_{3} \mathrm{Te}_{16}$
}

\author{
G. M. Pang,${ }^{1}$ M. Smidman,${ }^{1, *}$ W. H. Jiao, ${ }^{2}$ L. Jiao, ${ }^{1}$ Z. F. Weng, ${ }^{1}$ W. \\ B. Jiang, ${ }^{1}$ C. Y. Guo,${ }^{1}$ Y. Chen,${ }^{1}$ G. H. Cao ${ }^{1,3}$ and H. Q. Yuan ${ }^{1,3, \dagger}$ \\ ${ }^{1}$ Center for Correlated Matter and Department of Physics, Zhejiang University, Hangzhou 310058, China \\ ${ }^{2}$ Department of Physics, Zhejiang University of Science and Technology, Hangzhou 310023, China \\ ${ }^{3}$ Collaborative Innovation Center of Advanced Microstructures, Nanjing 210093, China
}

(Dated: June 11, 2018)

\begin{abstract}
We report an investigation of the London penetration depth $\Delta \lambda(T)$ on single crystals of the layered superconductor $\mathrm{Ta}_{4} \mathrm{Pd}_{3} \mathrm{Te}_{16}$, where the crystal structure has quasi-one-dimensional characteristics. A linear temperature dependence of $\Delta \lambda(T)$ is observed for $T \ll T_{c}$, in contrast to the exponential decay of fully gapped superconductors. This indicates the existence of line nodes in the superconducting energy gap. A detailed analysis shows that the normalized superfluid density $\rho_{s}(T)$, which is converted from $\Delta \lambda(T)$, can be well described by a multigap scenario, with nodes in one of the superconducting gaps, providing clear evidence for nodal superconductivity in $\mathrm{Ta}_{4} \mathrm{Pd}_{3} \mathrm{Te}_{16}$.
\end{abstract}

*msmidman@zju.edu.cn

† hqyuan@zju.edu.cn 


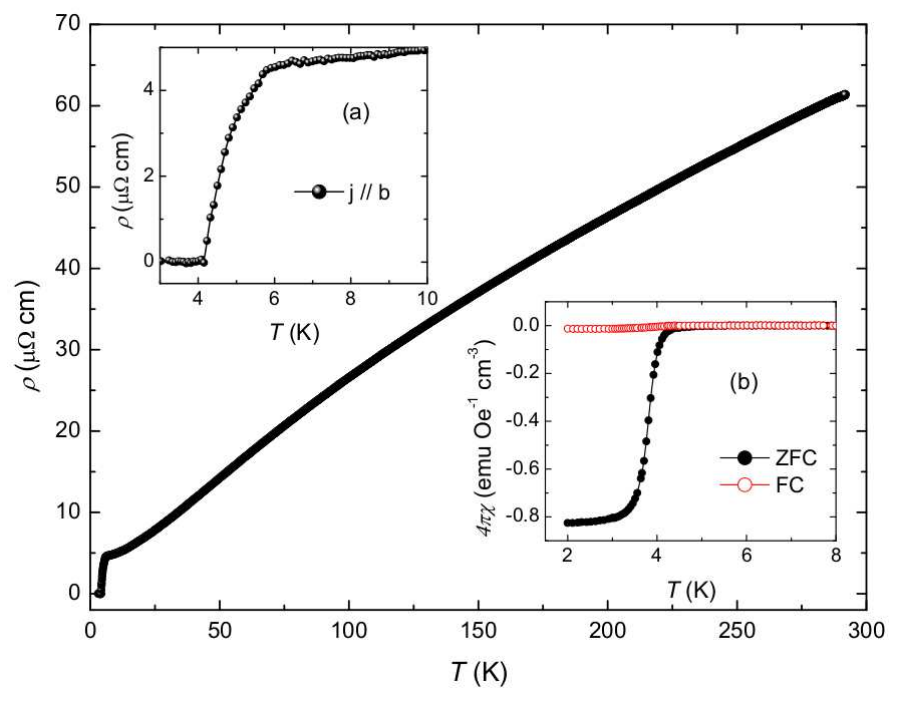

FIG. 1. The temperature dependence of the electrical resistivity $\rho(T)$ of $\mathrm{Ta}_{4} \mathrm{Pd}_{3} \mathrm{Te}_{16}$ from $292 \mathrm{~K}$ down to around $3 \mathrm{~K}$. The insets show the behavior of (a) $\rho(T)$ and (b) the magnetic susceptibility $4 \pi \chi(T)$ at low temperatures, where superconducting transitions are clearly observed.

\section{INTRODUCTION}

Recently the layered Pd-based ternary chalcogenides have attracted much research interest. New compounds with similar crystal structures were discovered, which opened up new opportunities to investigate the relationship between superconductivity and reduced crystal dimensionality [1-3]. Unusual superconducting properties were revealed in $\mathrm{Ta}_{2} \mathrm{Pd}_{x} \mathrm{~S}_{5}(x \lesssim 1.0)$ and $\mathrm{Nb}_{2} \mathrm{Pd}_{0.81} \mathrm{~S}_{5}$, where extremely large upper critical fields of $\mu_{0} \mathrm{H}_{c 2}(0)=31 \mathrm{~T}$ and $\mu_{0} \mathrm{H}_{c 2}(0)=37 \mathrm{~T}$ were observed respectively, both of which are almost twice the size of the Pauli limiting field $\left(H_{P}\right)[4,5]$. These are reminiscent of the quasi-one-dimensional (Q1D) organic compounds (TMTSF) $2 X$ (TMTSF = tetramethyltetraselenafulvalene, $X=\mathrm{PF}_{6}, \mathrm{ClO}_{4}$ ), which are believed to be unconventional superconductors $[6,7]$. Moreover, subsequent specific heat measurements in $\mathrm{Nb}_{2} \mathrm{Pd}\left(\mathrm{S}_{1-x} \mathrm{Se}_{x}\right)$ and $\mathrm{Ta}_{2} \mathrm{PdSe}_{5}$ show a slight deviation from the typical behavior of single band $s$-wave superconductors, which likely indicates multi-band superconductivity in these systems $[8,9]$.

A new layered Pd-based ternary compound $\mathrm{Ta}_{4} \mathrm{Pd}_{3} \mathrm{Te}_{16}$ was found to exhibit superconductivity at $T_{c} \approx 4.6 \mathrm{~K}$ [10]. It possesses a layered crystal structure as well as quasi-one-dimensional (Q1D) characteristics, with chains running along the $b$ axis. Band structure calculations for $\mathrm{Ta}_{4} \mathrm{Pd}_{3} \mathrm{Te}_{16}$ reveal that its Fermi surface consists of four branches, including two one-dimensional nested sheets, a two-dimensional cylindrical sheet and a three-dimensional one, which drives this compound to be an anisotropic but three-dimensional metal [11]. This is also consistent with the results of upper critical field measurements where a moderate anisotropy of $\mu_{0} H_{c 2}$ was observed and the coherence lengths along all three axes are much larger than the interchain distance [12]. High field measurements uncover a quasi-linear magnetoresistance without any sign of saturation up to about $50 \mathrm{~T}$, as well as a violation of Kohler's rule, indicating the existence of charge density wave (CDW) fluctuations in this compound [13], which has also been suggested from scanning tunneling spectroscopy (STS) experiments [14]. Meanwhile, there have also been various studies to characterize the superconducting order parameter, which could give the crucial information about the pairing mechanism. However, no firm conclusions have been reached on the nature of the gap structure. Evidence for nodal superconductivity came from thermal conductivity measurements, where in zero field there is a significant residual value of $\kappa(T) / T$ at zero temperature. Furthermore, there is a rapid increase of $\kappa(H) / T$ in an applied magnetic field, which is very similar to the behavior of $d$-wave superconductors [15]. This scenario is supported by electronic specific heat results, which show power law behavior at low temperatures and a non-linear field dependence of the Sommerfeld coefficient $\gamma(H)$ [12]. On the other hand, different results were obtained from STS measurements, where a BCS-like gap structure was reported by one group, whereas another report gave an indication of a highly anisotropic gap structure with gap minima or nodes $[14,16]$. Therefore, due to the discrepancies between different measurements of the order parameter of $\mathrm{Ta}_{4} \mathrm{Pd}_{3} \mathrm{Te}_{16}$, further measurements at lower temperatures which are sensitive to low energy excitations are badly needed.

Here, we report the temperature dependence of the London penetration depth $\Delta \lambda(T)$ of single crystals of 


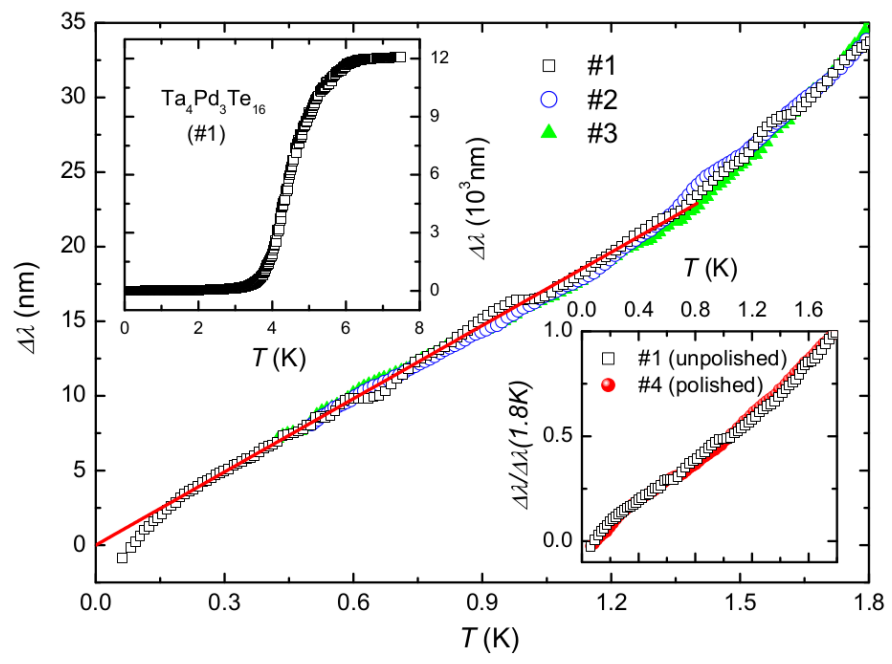

FIG. 2. Low temperature behavior of the London penetration depth $\Delta \lambda(T)$ for three single crystals of $\mathrm{Ta}_{4} \mathrm{Pd}_{3} \mathrm{Te}_{16}$. The solid red line shows the linear decrease of $\Delta \lambda(T)$ below around $1.4 \mathrm{~K}$. The upper inset shows the temperature dependence of $\Delta \lambda(T)$ up to above $T_{c}$. The lower inset shows a comparison between the low temperature behavior of an unpolished sample (\#1) and a sample where the surface was polished $(\# 4)$, normalized by the respective values at $1.8 \mathrm{~K}$.

$\mathrm{Ta}_{4} \mathrm{Pd}_{3} \mathrm{Te}_{16}$. A combined analysis of our $\Delta \lambda(T)$ measurements, the derived superfluid density $\rho(T)$ and the previous specific heat results show consistent evidence for multi-band superconductivity in $\mathrm{Ta}_{4} \mathrm{Pd}_{3} \mathrm{Te}_{16}$ with nodes in at least one of the gaps.

\section{METHODS}

Single crystal samples were synthesized by a self-flux method [10], and characterized using both electrical resistivity and magnetic susceptibility measurements. The temperature dependence of the resistivity was measured using the standard four-probe method from room temperature down to about $3 \mathrm{~K}$, while the magnetization measurements were performed by utilizing a SQUID magnetometer (MPMS-5T) from $8 \mathrm{~K}$ to about $2 \mathrm{~K}$ with both field-cooling (FC) and zero-field-cooling (ZFC) under a small applied magnetic field of 10 Oe.

By utilizing a tunnel-diode-oscillator (TDO) based technique [17], precise measurements of London penetration depth $\Delta \lambda(T)$ were carried out in a ${ }^{3} \mathrm{He}$ cryostat down to $0.45 \mathrm{~K}$, and in a dilution refrigerator with a base temperature of about $0.06 \mathrm{~K}$. Due to the flat needle like shape of the crystals, samples were cut into typical sizes of $(250-450) \times$ $(250-450) \times(50-150) \mu m^{3}$ with the plane being parallel to the chain direction. The sample was mounted on a sapphire rod so as to be inserted into the coil without any contact. The operating frequency of the TDO was about $7 \mathrm{MHz}$ in the ${ }^{3} \mathrm{He}$ system and $9 \mathrm{MHz}$ in the dilution refrigerator, with a noise level as low as $0.1 \mathrm{~Hz}$, by steadily controlling the temperature of the coil and electrical circuit independently. The sample experienced a very small ac field induced by the coil of about $20 \mathrm{mOe}$ along the $c^{*}$ direction, which is much smaller than the lower critical field $H_{c 1}$, ensuring that the sample was always in the Meissner state during the measurements. As a result, the measured frequency shift $\Delta f(T)$ can be considered to be proportional to the change of the London penetration depth in the $a^{*} b$ plane with $\Delta \lambda(T)=\mathrm{G} \Delta f(T)$, where the calibration constant $G$ is solely dependent on the sample and coil geometry [18].

\section{RESULTS}

The electrical resistivity $[\rho(T)]$ and magnetic susceptibility are displayed in Fig. 1. Metallic behavior of $\rho(T)$ is shown in the normal state, with a residual resistivity of $4.5 \mu \Omega \mathrm{cm}$, just before entering the superconducting state, with zero resistivity being reached at $4.1 \mathrm{~K}$. This gives rise to a large mean free path of $281 \mathrm{~nm}$ following the method in Ref.19, by using a coherence length of $\xi_{0}=10 \mathrm{~nm}$ and Sommerfeld coefficient $\gamma_{n}=51.2 \mathrm{~mJ} \mathrm{~mol}^{-1} \mathrm{~K}^{-2}$ [12]. This calculated mean free path is much larger than the coherence length. A superconducting transition is also observed in the magnetic susceptibility measurements, with a midpoint of the transition at around $3.8 \mathrm{~K}$. These results indicate 

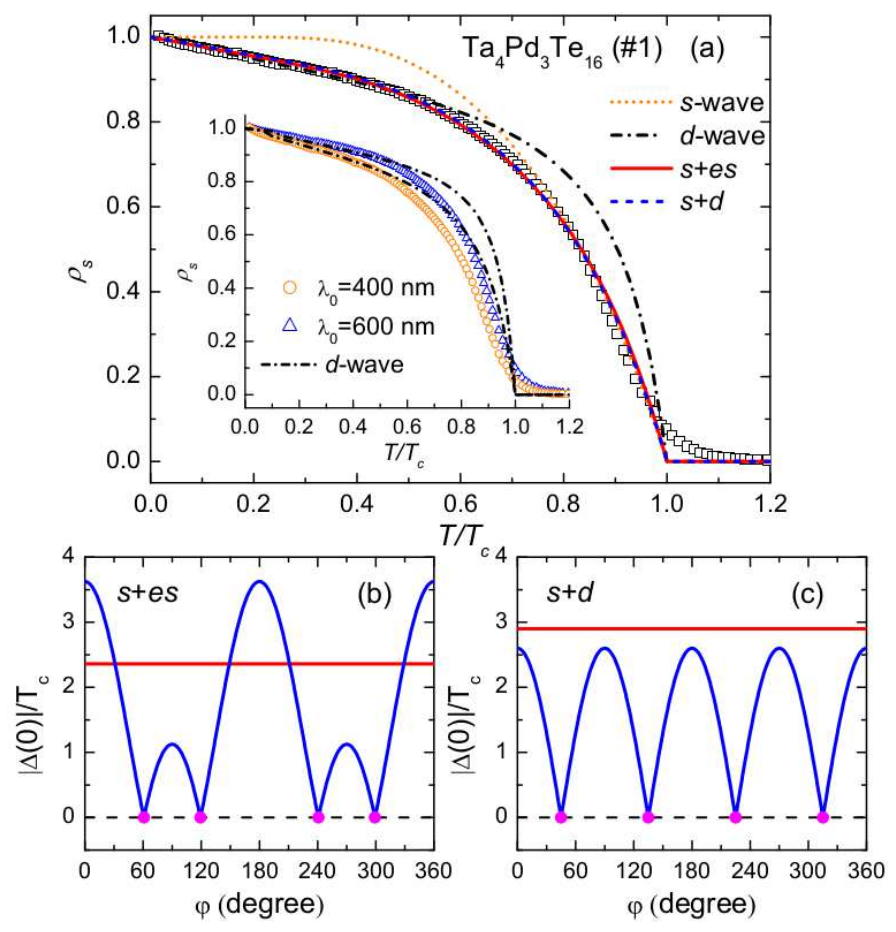

FIG. 3. (a) The temperature dependence of the normalized superfluid density $\rho_{s}(T)$ for sample \#1 of $\mathrm{Ta}_{4} \mathrm{Pd}_{3} \mathrm{Te}_{16}$, using $\lambda(0)=492 \mathrm{~nm}$. The lines show the results from fitting various models. The inset shows $\rho_{s}(T)$ for two different values of $\lambda(0)$, where dashed lines show fits to a $d$-wave model. The bottom panels show the angular dependence of the amplitudes of the two gaps at zero temperature from the fitted (b) $s+e s$ and (c) $s+d$ models, where the solid dots represent the nodal directions.

that $\mathrm{Ta}_{4} \mathrm{Pd}_{3} \mathrm{Te}_{16}$ is a superconductor in the clean limit.

As shown in Fig. 2, the temperature dependence of the London penetration depth shift $\Delta \lambda(T)$ for various single crystals was measured from $8 \mathrm{~K}$ down to $0.06 \mathrm{~K}$, which exhibits similar reproducible behavior. A clear superconducting transition is observed in $\Delta \lambda(T)$, with $T_{c}=3.9 \mathrm{~K}$ determined from the endpoint of the transition, which was used in the subsequent calculations. We note that in both the resistivity and TDO based measurements, there is an onset of superconductivity at higher temperatures of around $6 \mathrm{~K}$, whereas the endpoints of the transitions are close to the $T_{c}$ values in the specific heat and dc magnetic susceptibility. Since the resistivity and frequency shift are much more sensitive to non-bulk superconductivity, these results suggest that there is the onset of filamentary superconductivity with a small volume fraction at higher temperatures, above the sharp onset of bulk superconductivity in the rest of the sample. The main panel displays the enlargement of the low temperature behavior of $\Delta \lambda(T)$, where a quasilinear temperature dependence is observed from $1.4 \mathrm{~K}$ down to about $0.2 \mathrm{~K}$, below which a small downturn in $\Delta \lambda(T)$ is observed. The temperature dependence of $\Delta \lambda(T)$ at $T \ll T_{c}$ is usually related to the low energy excitations, which is determined by the superconducting gap structure. In the case of nodeless weakly coupled s-wave superconductors, $\Delta \lambda(T)$ regularly exhibits an exponential decrease below around $T_{c} / 3$, due to the absence of low energy excitations. Whereas in other materials, such as the cuprate and heavy fermion superconductors where there are often nodes in the gap, a power law temperature dependence of $\Delta \lambda(T)$ is usually observed and in particular, when $\Delta \lambda(T) \sim \mathrm{T}$ at low temperatures, there is a strong indication of line nodes [20,21]. Therefore, the clear observation of linear behavior at low temperatures in the penetration depth measurements is evidence against fully-gapped superconductivity in $\mathrm{Ta}_{4} \mathrm{Pd}_{3} \mathrm{Te}_{16}$, and suggests that the superconducting gap has line nodes. We note that the small downturn below $0.2 \mathrm{~K}$ is consistently observed in different samples, including when the sample is polished to avoid any extrinsic effect from the as-grown surface (lower inset of Fig. 2). The overall size of the downturn of the signal is only around $0.04 \%$ of the overall frequency shift from above $T_{c}$ and therefore this may correspond to the presence of a very small impurity phase, which is not likely to significantly affect the measurements at higher temperatures.

To get further insight into the superconducting pairing symmetry of $\mathrm{Ta}_{4} \mathrm{Pd}_{3} \mathrm{Te}_{16}$, the normalized superfluid density $\rho_{s}(T)$, calculated using $\rho_{s}(T)=\lambda^{2}(0) / \lambda^{2}(T)$ with $\lambda(T)=\lambda(0)+\Delta \lambda(T)$, is plotted in Fig. 3(a). The value of $\lambda(0)$ was derived from solving $\mu_{0} H_{c 1}=\left(\Phi_{0} / 4 \pi \lambda^{2}\right)[\ln (\lambda / \xi)+0.5]$, where $\Phi_{0}$ is the magnetic flux quantum. By using the parameters $\mu_{0} H_{c 2}(0)=3.3 \mathrm{~T}$ and $\mu_{0} H_{c 1}(0)=2.99 \mathrm{mT}$ [12], the value of $\lambda(0)=492 \mathrm{~nm}$ was estimated. The inset of Fig. 3(a) also shows $\rho_{s}(T)$ for a change in $\lambda(0)$ of $\pm \sim 20 \%$ and the data show similar behavior. The temperature 


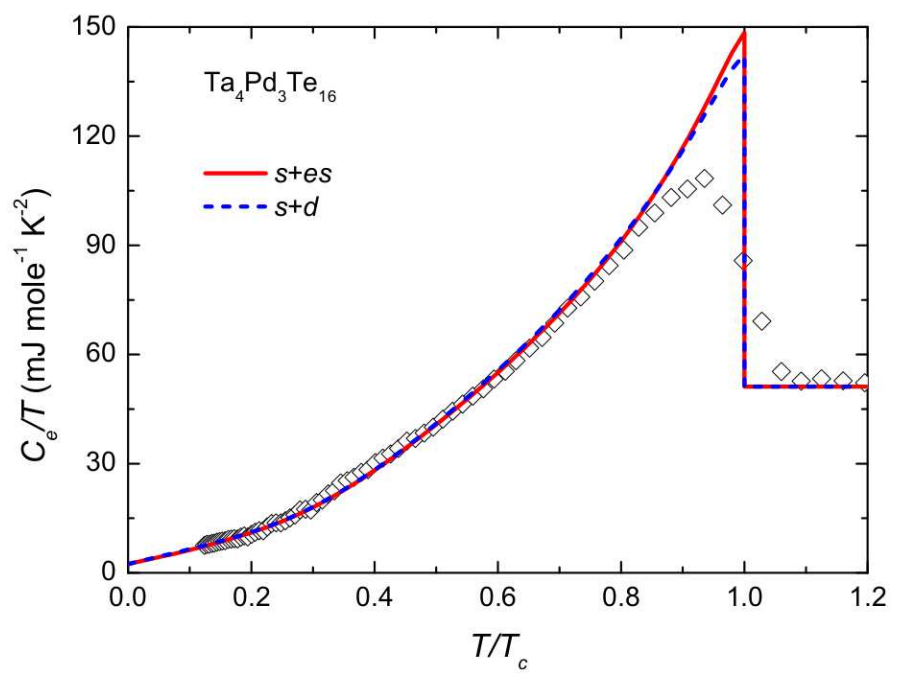

FIG. 4. Electronic specific heat of a single crystal of $\mathrm{Ta}_{4} \mathrm{Pd}_{3} \mathrm{Te}_{16}$ from Ref. 12 . The lines show the fits to the $s+e s$ and $s+d$ models, which overlap well with the experimental data.

dependence of $\rho_{s}(T)$ solely depends on its Fermi surface and superconducting gap structure. For a given gap function $\Delta_{k}$, the normalized superfluid density $\rho_{s}(T)$ is calculated using:

$$
\rho_{\mathrm{S}}(T)=1+2\left\langle\int_{\Delta_{k}}^{\infty} \frac{E \mathrm{~d} E}{\sqrt{E^{2}-\Delta_{k}^{2}}} \frac{\partial f}{\partial E}\right\rangle_{\mathrm{FS}},
$$

where $f(E, T)=[1+\exp (E / T)]^{-1}$ is the Fermi distribution function with the Boltzmann constant defined as $k_{B}=1$, and $\langle\ldots\rangle_{\mathrm{FS}}$ denotes the integration over the Fermi surface. The superconducting gap function is given by $\Delta_{k}(T)=$ $\Delta(T) g_{k}(\phi)$. Here, $g_{k}(\phi)$ is a dimensionless function that determines the angular dependence of the gap and $\phi$ is the azimuthal angle. $\Delta(T)$ describes the temperature dependence of the gap, which is approximated by:

$$
\Delta(T)=\Delta(0) \tanh \left\{1.82\left[1.018\left(T_{c} / T-1\right)\right]^{0.51}\right\},
$$

where the parameter which characterizes the zero temperature gap magnitude $\Delta(0)$ is the only adjustable parameter [22].

Several forms of $g_{k}(\phi)$ were used to fit the experimental data, as displayed in Fig. 3(a). Firstly, a single band $s$-wave model with $g_{k}(\phi)=1$ fails to describe the data at low temperatures, although the high temperature part above $0.7 T_{c}$ is fitted quite well. When the superconducting gap is nodeless, $\rho_{s}(T)$ is always expected to flatten at $T \ll T_{c}$, but no such saturation is observed in the experimental data and $\rho_{s}(T)$ continues to increase with decreasing temperature. The observation of a linear temperature dependence of $\Delta \lambda(T)$ and $\rho_{s}(T)$ provides evidence for the existence of line nodes in the superconducting energy gap, as in the case of the $d$-wave superconductivity of the cuprates. Consequently, a $d$-wave model with $g_{k}(\phi)=\cos 2 \phi$ was fitted, which successfully describes $\rho_{s}(T)$ at low temperatures but significantly deviates in the high temperature region, implying that a single band $d$-wave model is also not a reasonable scenario. Although the derived $\rho_{s}(T)$ data will be affected by uncertainties in both the calibration constant $G$ and $\lambda(0)$, as displayed in the inset, upon changing $\lambda(0)$ by $\pm \sim 20 \%$, a $d$-wave model is still unable to account for the data.

Due to the presence of multiple Fermi surface sheets from theoretical calculations [11], we also fitted various twoband models. In a phenomenological two-band model, the total superfluid density $\rho_{s}(T)$ can be obtained from a linear combination of different two components:

$$
\rho_{\mathrm{s}}(T)=\alpha \rho_{\mathrm{s}}^{1}\left(\Delta_{k}^{1}, T\right)+(1-\alpha) \rho_{\mathrm{s}}^{2}\left(\Delta_{k}^{2}, T\right),
$$

where $\Delta_{k}^{i}(i=1,2)$ represent the superconducting gap functions of the two components and $\alpha$ is the relative weight for $\rho_{s}^{1}$. Since a linear temperature dependence of $\rho_{s}(T)$ is clearly observed at $T \ll T_{c}$, which can not be reproduced by a calculation with two isotropic nodeless gaps, both $s+e s$ and $s+d$ models were fitted to the experimental data. In the $s+e s$ model there is one isotropic gap and an anisotropic $s$-wave (es) gap, with a gap angular dependence given by $g_{k}(\phi)=1+r \cos 2 \phi$, where $r$ characterizes the gap anisotropy [12, 16, 23]. It can be seen that the energy gap is always 
nodeless for $r<1$, while for $r>1$, it goes to zero along $\phi=0.5 \arccos (-1 / r)$, where the accidental nodes are located. We note that a single band anisotropic $s$-wave model was previously reported to be unable to account for the specific heat data [12]. On the other hand the $s+d$ model has an isotropic $s$-wave gap as well as a $d$-wave gap, and this model was also applied in the analysis of STS and specific heat data, where the nodal $d$-wave component was one possibility for explaining the deviation from isotropic, fully-gapped behavior $[12,16]$. As shown in Fig. 3(a), both models can well fit the experimental data across the whole temperature range. For the $s+e s$ model, the fitting parameters are $\Delta_{s}(0)=2.36 T_{c}, \Delta_{e s}(0)=1.25 T_{c}$ and $r=1.9$, with a weighting of $\alpha=0.3$ for the isotropic $s$-wave component. For the $s+d$ model, the fitted values are $\Delta_{s}(0)=2.9 T_{c}, \Delta_{d}(0)=2.6 T_{c}$ and $\alpha=0.6$. The angular dependence of the gap structures for the fitted models are displayed in Fig. 3 (b) and (c), where the value of $r=1.9$ indicates that for the $s+e s$ model, there are nodes in the anisotropic gap. It should be noted that the linear temperature dependence of $\rho_{s}(T)$ at low temperatures can not be reproduced by an $s+e s$ model with $r<1$, where both superconducting gaps are fully open. In that case, due to a nodeless superconducting gap structure, $\Delta \lambda(T)$ and the derived $\rho_{s}(T)$ always become flat below a certain temperature depending on the value of the gap magnitude, whereas no such saturation is observed in the measurements, even down to $0.06 \mathrm{~K}\left(\sim 0.015 T_{c}\right)$.

In addition, the $s+e s$ and $s+d$ models were also fitted to the previously reported electronic specific heat data from Ref. 12, as displayed in Fig. 4. The entropy $S$ in the superconducting state can be expressed as [24]:

$$
S=-\frac{3 \gamma}{\pi^{3}} \int_{0}^{2 \pi} \int_{0}^{\infty}[f \ln f+(1-f) \ln (1-f)] \mathrm{d} \varepsilon \mathrm{d} \phi
$$

where $\varepsilon=\sqrt{E^{2}-\Delta_{k}^{2}(T)}$ and $\Delta_{k}(T)$ follows the same expression used in the superfluid density analysis. In the superconducting state, the superconducting electronic specific heat is derived from $C_{e}=T \mathrm{~d} S / \mathrm{d} T$. Both the $s+e s$ and $s+d$ models can well describe the data, taking into account a residual contribution to $C_{e} / T$ of $\gamma_{0}=2.4 \mathrm{~mJ} \mathrm{~mol}^{-1}$ $\mathrm{K}^{-2}$. The fitted parameters are $\Delta_{s}(0)=2.1 T_{c}, \Delta_{e s}(0)=1.16 T_{c}, \alpha=0.53$ and $\mathrm{r}=2.0$ for the $s+e s$ model and $\Delta_{s}(0)=2.45 T_{c}, \Delta_{d}(0)=2.05 T_{c}$, and $\alpha=0.48$ for the $s+d$ model. Since the specific heat is sensitive to excitations along all directions but $\rho_{s}(\mathrm{~T})$ only probes directions perpendicular to the applied field, this may account for the small differences between the fitting parameters from the two techniques.

Therefore, both two-band $s+e s$ and $s+d$ models can reasonably account for the penetration depth and specific heat data, so it is difficult to distinguish between these two scenarios on the basis of our measurements. The primary result of our present study is the linear behavior of $\Delta \lambda(\mathrm{T})$ at low temperatures, which strongly suggests the existence of line nodes in the gap structure. However, in the case of the $s+d$ model, the $s$-wave and $d$-wave instabilities will generally be expected to have different transition temperatures and therefore a split superconducting transition would be anticipated [25]. It has also been noted that the nodal superconducting gap structure is expected to be more robust in $d$-wave superconductors than in the extended $s$-wave cases, where the accidental nodes can be easily lifted by the effect such as disorder [26]. Therefore, it maybe helpful to study samples where random defects are introduced, for example by electron irradiation. On the other hand, theoretical calculations indicate that there are complex anisotropic Fermi sheets in $\mathrm{Ta}_{4} \mathrm{Pd}_{3} \mathrm{Te}_{16}$, and suggest the system is far away from a magnetic instability [11]. In that sense, the complex Fermi surface maybe play an important role in leading to the anisotropic interactions which give rise to nodes.

\section{CONCLUSION}

To summarize, we have precisely measured the temperature dependence of the change of the London penetration depth $\Delta \lambda(T)$ for the newly discovered layered superconductor $\mathrm{Ta}_{4} \mathrm{Pd}_{3} \mathrm{Te}_{16}$ using a TDO method. Linear behavior of $\Delta \lambda(T)$ is clearly observed at low temperatures, as well as in the corresponding superfluid density $\rho_{s}(T)$, which can be successfully described in terms of either a phenomenological two-band $s+e s$ or $s+d$ model, in line with our reanalysis of the previous specific heat results. Our findings show a distinct discrepancy from the behavior of fully-gapped superconductors and provide strong evidence for nodal superconductivity in $\operatorname{Ta}_{4} \mathrm{Pd}_{3} \mathrm{Te}_{16}$.

\section{ACKNOWLEDGMENTS}

We thank X. Lu, C. Cao and D. F. Agterberg for helpful discussions and suggestions. This work was supported by the National Key R\&D Program of China (No. 2016YFA0300202,No. 2017YFA0303100,), the National Natural 
Science Foundation of China (No. 11474251), and the Science Challenge Project of China (No. TZ2016004).

[1] Zhang Q R, Rhodes D, Zeng B, Besara T, Siegrist T, Johannes M D and Balicas L 2013 Anomalous metallic state and anisotropic multiband superconductivity in $\mathrm{Nb}_{3} \mathrm{Pd}_{0.7} \mathrm{Se}_{7}$ Phys. Rev. B 88024508

[2] Yu H Y, Zuo M, Zhang L, Tan S, Zhang C J and Zhang Y H 2013 Superconducting Fiber with Transition Temperature up to $7.43 \mathrm{~K}$ in $\mathrm{Nb}_{2} \mathrm{Pd}_{x} \mathrm{~S}_{5-\delta}(0.6<\mathrm{x}<1)$ J. Am. Chem. Soc.135 12987

[3] Jiao W H, He L P, Liu Y, Xu X F, Li Y K, Zhang C H, Zhou N, Xu Z A, Li S Y and Cao G H 2016 Superconductivity in $\mathrm{Ta}_{3} \mathrm{Pd}_{3} \mathrm{Te}_{14}$ with quasi-one-dimensional $\mathrm{PdTe}_{2}$ chains Sci. Rep. 621628

[4] Lu Y F, Takayama T, Bangura A F, Katsura Y, Hashizume D and Takagi H 2013 Superconductivity at 6 K and the Violation of Pauli Limit in $\mathrm{Ta}_{2} \mathrm{Pd}_{x} \mathrm{~S}_{5}$ J. Phys. Soc. Jpn. 83023702

[5] Zhang Q, Li G, Rhodes D, Kiswandhi A, Besara T, Zeng B, Sun J, Siegrist T, Johannes M D and Balicas L 2013 Superconductivity with extremely large upper critical fields in $\mathrm{Nb}_{2} \mathrm{Pd}_{0.81} \mathrm{~S}_{5}$ Sci. Rep. 31446

[6] Lee I J, Naughton M J, Danner G M and Chaikin P M 1997 Anisotropy of the Upper Critical Field in (TMTSF) ${ }_{2} \mathrm{PF}_{6}$ Phys. Rev. Lett. 783555

[7] Oh J I and Naughton M J 2004 Magnetic Determination of $\mathrm{H}_{c 2}$ under Accurate Alignment in $(\mathrm{TMTSF})_{2}$ ClO 4 Phys. Rev. Lett. 92067001

[8] Zhang J, Dong J K, Xu Y, Pan J, He L P, Zhang L J and Li S Y 2015 Superconductivity at 2.5 K in the new transition-metal chalcogenide $\mathrm{Ta}_{2} \mathrm{PdSe}_{5}$ Supercond. Sci. Technol. 28115015

[9] Niu C Q et al. 2013 Effect of selenium doping on the superconductivity of $\mathrm{Nb}_{2} \mathrm{Pd}\left(\mathrm{S}_{1-x} \mathrm{Se}_{x}\right)$ Phys. Rev. B 88 104507

[10] Jiao W H, Tang Z T, Sun Y L, Liu Y, Tao Q, Feng C M, Zeng Y W, Xu Z A and Cao G H 2014 Superconductivity in a layered $\mathrm{Ta}_{4} \mathrm{Pd}_{3} \mathrm{Te}_{16}$ with $\mathrm{PdTe}_{2}$ chains J. Am. Chem. Soc.136 1284

[11] Singh D J 2014 Multiband superconductivity of $\mathrm{Ta}_{4} \mathrm{Pd}_{3} \mathrm{Te}_{16}$ from Te $p$ states Phys. Rev. B 90144501

[12] Jiao W H, Liu Y, Li Y K, Xu X F, Bao J K, Feng C M, Li S Y, Xu Z A and Cao G H 2015 Multiband superconductivity in $\mathrm{Ta}_{4} \mathrm{Pd}_{3} \mathrm{Te}_{16}$ with anisotropic gap structure J. Phys.: Condens. Mat. 27325701

[13] Xu X F et al. 2015 Quasi-linear magnetoresistance and the violation of Kohler's rule in the quasi-one-dimensional $\mathrm{Ta}_{4} \mathrm{Pd}_{3} \mathrm{Te}_{16}$ superconductor J. Phys.: Condens. Mat. 27335701

[14] Fan Q et al. 2015 Scanning tunneling microscopy study of superconductivity, magnetic vortices, and possible charge-density wave in $\mathrm{Ta}_{4} \mathrm{Pd}_{3} \mathrm{Te}_{16}$ Phys. Rev. B 91104506

[15] Pan J, Jiao W H, Hong X C, Zhang Z, He L P, Cai P L, Zhang J, Cao G H and Li S Y 2015 Nodal superconductivity and superconducting dome in the layered superconductor $\mathrm{Ta}_{4} \mathrm{Pd}_{3} \mathrm{Te}_{16}$ Phys. Rev. B 92180505

[16] Du Z Y, Fang D L, Wang Z Y, Li Y F, Du G, Yang H, Zhu X Y and Wen H H 2015 Anisotropic Superconducting Gap

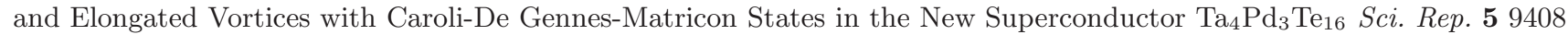

[17] Van Degrift C T 1975 Tunnel diode oscillator for 0.001 ppm measurements at low temperatures Rev. Sci. Instrum. 46 599

[18] Prozorov R, Giannetta R W, Carrington A and Araujo-Moreira F M 2000 Meissner-London state in superconductors of rectangular cross section in a perpendicular magnetic field Phys. Rev. B 62115

[19] Orlando T P, McNiff E J, Foner S and Beasley M R 1979 Critical fields, Pauli paramagnetic limiting, and material parameters of $\mathrm{Nb}_{3} \mathrm{Sn}$ and $\mathrm{V}_{3} \mathrm{Si}$ Phys. Rev. B 194545

[20] Hardy W N, Bonn D A, Morgan D C, Liang R X and Zhang K 1993 Precision measurements of the temperature dependence of $\lambda$ in $\mathrm{YBa}_{2} \mathrm{Cu}_{3} \mathrm{O}_{6.95}$ : strong evidence for nodes in the gap functionPhys. Rev. Lett. 703999

[21] Chia E E M, Van Harlingen D J, Salamon M B, Yanoff B D, Bonalde I and Sarrao J L 2003 Nonlocality and strong coupling in the heavy fermion superconductor CeCoIn : a penetration depth study Phys. Rev. B 67014527

[22] Carrington A and Manzano F 2003 Magnetic penetration depth of MgB 2 Physica C 385205

[23] Lin J -Y, Hsieh Y S, Chareev D A, Vasiliev A N, Parsons Y and Yang H D 2011 Coexistence of isotropic and extended $s$-wave order parameters in FeSe as revealed by low-temperature specific heat Phys. Rev. B 84 220507(R)

[24] Bouquet F, Wang Y, Fisher R A, Hinks D G, Jorgensen J D, Junod A and Phillips N E 2001 Phenomenological two-gap model for the specific heat of $\mathrm{MgB}_{2} E P L \mathbf{5 6} 856$

[25] Sigrist M 2000 Broken time reversal symmetry in unconventional superconductors Physica C 341-348 695-698

[26] Reid J -Ph et al. 2012 From $d$-wave to $s$-wave pairing in the iron-pnictide superconductor (Ba,K)Fe $\mathrm{B}_{2} \mathrm{Bs}_{2}$ Supercond. Sci. Technol. 25084013 